\title{
A Single-mode, High Index-contrast, Lead Silicate Glass Fibre with High Nonlinearity, Broadband Near-zero Dispersion at Telecommunication Wavelengths
}

\author{
X. Feng, G. M. Ponzo, F. Poletti, A. Camerlingo, F. Parmigiani, M. N. Petrovich, P. Petropoulos, N. M. \\ White, W. H. Loh, D. J. Richardson \\ Optoelectronic Research Centre, University of Southampton, SO17 1BJ, United Kingdom, \\ xif@orc.soton.ac.uk
}

\begin{abstract}
We report on the design, fabrication and characterization of a single-mode $W$-type leadsilicate glass fibre with flattened and near-zero dispersion profile at telecom wavelengths and high nonlinearity of $820 W^{-1} \mathrm{~km}^{-1}$ at $1.55 \mu \mathrm{m}$.
\end{abstract}

\section{Introduction}

Holey Fibre (HF) technology ${ }^{1}$ enables unprecedented control over fibre transmission properties, such as nonlinearity, dispersion, and single-mode operation, by tailoring the structural parameters of the cladding, i.e., the hole-to-hole spacing and the hole size. Hence, it has allowed substantial improvements in the development of single-mode, high nonlinearity fibres with tailored dispersion profiles (in particular with flattened and near-zero values), for photonic device applications, such as all-optical switches, wavelength converters, optical parametric amplifiers and oscillators, and supercontinuum generators. Several high-nonlinearity and dispersion-flattened air/glass HFs have been demonstrated using either silica ${ }^{2}$ or non-silica ${ }^{3}$ glass as the host material. The ability to accurately control the structure in fibres with $\mu \mathrm{m}$ scale hole size and spacing, represents a major challenge in terms of fibre fabrication. Indeed, the presence of air holes significantly increases the difficulty to accurately match a structural design target because of unavoidable smallscale longitudinal variations of the cross section that arise due to the complex interdependence between temperature, surface tension and internal pressure within the holes. As a result, precise dispersion control to the $\mathrm{ps} / \mathrm{nm} / \mathrm{km}$ level is extremely hard to achieve. In addition, due to the nature of single material fibres, it is normally not feasible to achieve single-mode operation, near-zero dispersion, and maximum nonlinearity simultaneously in a HF. To solve these technical issues, in this work we propose a new all-solid fibre concept based on the use of three lead silicate glasses arranged in a W-type index profile. The fabricated fibre presents a flattened and near-zero dispersion profile, a high nonlinearity of $820 \mathrm{~W}^{-1} \mathrm{~km}^{-1}$, and a loss of $2.1 \mathrm{~dB} / \mathrm{m}$ in the $1.55 \mu \mathrm{m}$ region.
Fibre design, fabrication and characterization

Dispersion flattened, single material HFs comprise an engineered holey cladding where wavelength-scale hole sizes produce a waveguide dispersion which approximately compensates for the glass dispersion in both value and slope in a given wavelength range. The large index contrast between air and the background material enables a strong waveguide dispersion contribution and hence flattened profiles even at wavelengths where the material dispersion is very large (and normal). This solution can be regarded as a 'structure'based route to tailor the fibre dispersion.

Similarly, a 'material'-based route to control the fibre dispersion also exists for conventional, non-microstructured optical fibres. The idea is that for any given core material, by choosing a cladding material with the same index as the effective index of the holey cladding in a dispersion flattened HF analogue, a similar total dispersion profile can be obtained at any one particular wavelength.

Although the 'structure'-based route has the advantage of requiring just one glass, there are inherent difficulties in achieving the desired air hole dimensions with accurate precision, as already mentioned. In this work, we explore the 'material'-based route and demonstrate for the first time a high index contrast fibre with dispersion flattened properties. As the core material we chose SF57 glass and searched for a suitable cladding glass in order to obtain a flattened and near-zero dispersion profile around $1.55 \mu \mathrm{m}$ in a step-index fibre structure. Schott SF57 glass is a commercial lead silicate glass with a refractive index $n$ of $1.80^{4}$ and a high nonlinear index $\mathrm{n}_{2}$ of $41 \times 10^{-20} \mathrm{~m}^{2} / \mathrm{W}$ at $1.55 \mu \mathrm{m}$. SF57 glass HFs have been studied in detail in terms of fibre design, fibre fabrication, and applications for nonlinear fibre optics ${ }^{5}$. 
Combining practical material constraints with the results from numerical simulations, we found that Schott LLF1 glass was an excellent option as a cladding medium. When the core diameter is reduced to wavelength-scale dimensions, the combination SF57-LLF1 provides a high enough index-contrast and hence a strong enough waveguide dispersion to compensate for the core material dispersion. As shown in Fig.1(a), such a step-index fibre with a core diameter $d_{0}$ of 1.64-1.68 $\mu \mathrm{m}$ has a low dispersion $D$ (absolute value $|D|$ less than $5 \mathrm{ps} / \mathrm{nm} / \mathrm{km}$ ) in the wavelength range between 1.45 and $1.65 \mu \mathrm{m}$. According to our calculations, however, this particular structure is predicted to support two modes, as shown in Fig.1(b).

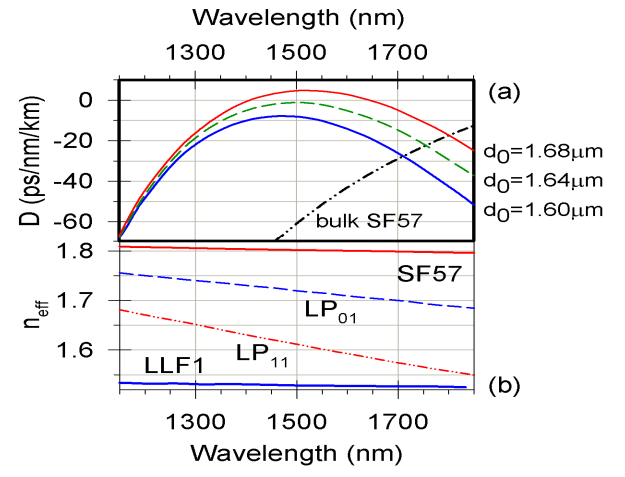

Fig. 1: Numerical simulation of (a) dispersion profile and (b) effective index $n_{\text {eff }}$ of $L P_{01}$ and $L P_{11}$ modes of a SF57-LLF1 step-index fibre

In order to obtain single-mode operation in such a step-index fibre, in analogy to the concept used in conventional W-type silica fibres with low index-contrast ${ }^{6}$, an outer cladding with a higher index than the effective index of the $\mathrm{LP}_{11}$ mode is introduced in the fibre structure. By choosing an appropriate diameter ratio for the first and second cladding, it is possible to impose a very high differential confinement loss on the $\mathrm{LP}_{11}$ mode, thus achieving effectively single mode operation for a broad wavelength range. For the second cladding we chose Schott SF6 glass $(n=1.76$ at $1.55 \mu \mathrm{m}$ ), and found that the resulting SF57LLF1-SF6 W-type fibre with $\mathrm{d}_{0}=1.68 \mu \mathrm{m}$ and $\mathrm{d}_{1}=7.4 \mu \mathrm{m}$ (see Fig.2), not only is single-moded but also has a dispersion profile very similar to the SF57-LLF1 step-index fibre with the same core diameter (shown in Fig.1(a)).

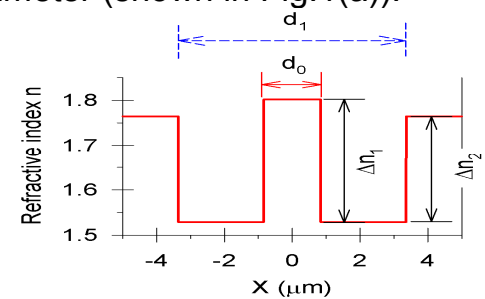

Fig. 2: Schematic of SF57-LLF1-SF6 W-type fibre
In order to realize such a fibre design in practice, the crucial issue is whether the three proposed glasses are thermally and chemically compatible with each other during the processes involved in the fibre fabrication, and in particular the fibre drawing. Fig.3 shows the measured viscosity curves of Schott SF57, LLF1 and SF6 glasses in the viscosity range $\left(10^{6.5}-10^{4}\right.$ poise) suitable for fibre drawing. As it can be seen, the three glasses have a relatively large viscosity mismatch, which obviously represents a fabrication challenge. Based on previous experience and on preliminary tests, we proved that with careful definition of the drawing conditions, fibres with good optical and mechanical properties could be nonetheless fabricated, as shown in the following.

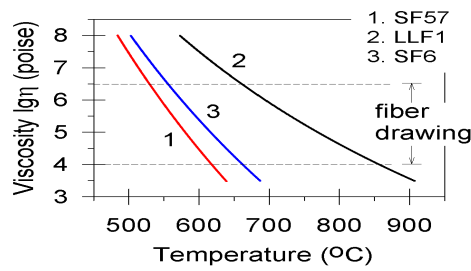

Fig. 3: Measured viscosity curves of Schott SF57, LLF1 and SF6 glasses

The W-type fibre was fabricated using the rod-in-tube method. The inner region was made from a $2.9 \mathrm{~mm}$ outer diameter (OD) SF57 rod inserted inside a LLF1 tube with an inner diameter (ID) of $3 \mathrm{~mm}$ and an OD of $13 \mathrm{~mm}$. The rod surface and both inner and outer surfaces of the tube were polished to optical quality. A SF57-LLF1 cane with $0.8 \mathrm{~mm}$ OD was then drawn from this assembly and inserted into a SF6 jacketing tube, from which the final fibre was drawn. Fig.4 shows the Scanning Electron Microscope (SEM) images of the cross-section of the fabricated fibre. The fibre has a core diameter of $1.63 \mu \mathrm{m}$ and a ratio of SF57 core diameter to LLF1 cladding diameter of 1:4.4, both of which exactly match the values in the initial preform and in the original target. This straightforward achievement of structural targets clearly represents an advantage of our all-solid fibre technology over the holey fibre counterpart.

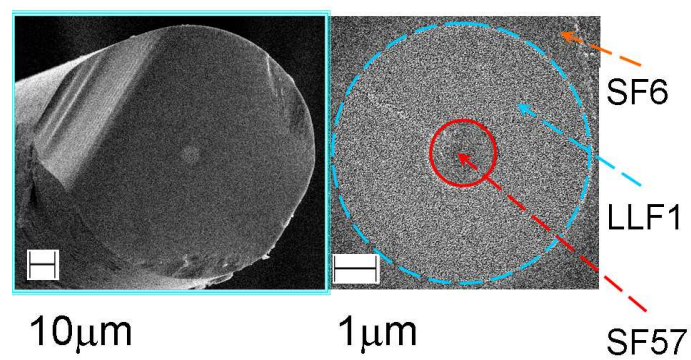

Fig. 4: SEM photos of W-fibre with $d_{0}$ of $1.63 \mu \mathrm{m}$

The propagation loss of the fabricated fibre was measured to be $2.1 \pm 0.2 \mathrm{~dB} / \mathrm{m}$ at $1.55 \mu \mathrm{m}$ 
using the cutback method. This is close to the bulk loss of commercial SF57 glass, $1.6 \pm 0.3 \mathrm{~dB} / \mathrm{m}$ at $1.53 \mu \mathrm{m}^{4}$. We expect this loss value can be reduced by further optimization of the fabrication process. The predicted effective single mode guidance was confirmed by analysing the near-field mode profiles of the fibre at $1.55 \mu \mathrm{m}$.

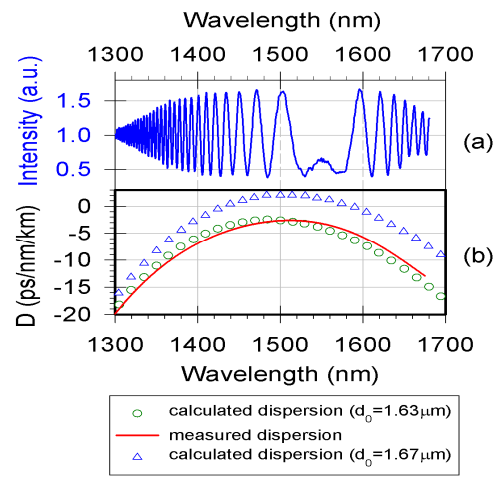

Fig. 5: (a) Observed interferometric pattern of one polarizations propagating through $\mathrm{W}$-fibre; (b) measured dispersion and calculated dispersion of Wfibre with core diameter $\mathrm{d}_{0}$ of $1.63 \& 1.67 \mu \mathrm{m}$

Despite the inherent difficulties in measuring the fibre's dispersion due to its low absolute value and short effective length, we obtained a very accurate and broadband measurement using a low-coherence Mach-Zehnder interferometric set-up and a supercontinuum source. High quality interferograms were measured for the two fundamental polarization states, one of which is shown in Fig. 5(a), from 1300 to 1680 $\mathrm{nm}$. The resulting dispersion curve, shown in Fig.5(b), is in excellent agreement with the numerical predictions, and shows a flattened profile at telecom wavelengths. In particular, at $\approx 1525 \mathrm{~nm}$ the dispersion slope is zero and $\mathrm{D}=-$ $2.6 \mathrm{ps} / \mathrm{nm} / \mathrm{km}$. Fig.5(b) also shows that by drawing a $2 \%$ larger fibre, a region of small and flat anomalous dispersion a few hundreds of $\mathrm{nm}$ wide can be achieved.

The fibre effective nonlinearity $\gamma$ at $1550 \mathrm{~nm}$ was measured with the dual $\mathrm{CW}$ method to be $820 \mathrm{~W}^{-1} \mathrm{~km}^{-1}$, in good agreement with a simulated value of $854 \mathrm{~W}^{-1} \mathrm{~km}^{-1}$ (effective area $\mathrm{A}_{\text {eff }} \approx 2 \mu \mathrm{m}^{2}$ ). Note that this value is 1.6 times higher than the predicted nonlinearity of a SF57 HF with zero dispersion and zero dispersion slope at $1.55 \mu \mathrm{m}^{5}$, which shows a second, substantial advantage of the present design as compared to the air/glass counterpart.

\section{Parametric wavelength conversion}

We tested the performance of the fabricated fibre as a broadband wavelength converter based on parametric four-wave-mixing (FWM) effects. A quasi CW pump at $1550 \mathrm{~nm}$, amplitude modulated with a 1:64 duty cycle and amplified, and a C-band tunable amplified CW signal were combined and free-space launched into a $2.2 \mathrm{~m}$ length of $\mathrm{W}$-fibre. The coupling efficiency at launch was $25 \%$ and the average pump and signal powers launched into the fibre were $17 \mathrm{dBm}$ and $2 \mathrm{dBm}$, respectively. Figs.6(a)\&(b) show the measured spectral traces for several input signal wavelengths and the resulting conversion efficiency (defined as the peak idler power over the average signal power). As can be seen, an impressive conversion efficiency of $\sim 0 \mathrm{~dB}$ was obtained throughout the $40 \mathrm{~nm}$ tuning range of our sources, in good agreement with numerical simulations.
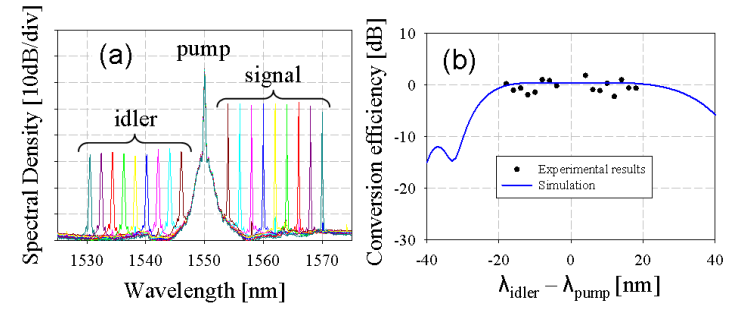

Fig. 6: (a) measured spectral traces of wavelength conversion in W-fibre; (b) measured and simulated idler's conversion efficiency

\section{Conclusions}

We have presented a new design concept for an all solid soft glass fibre which achieves effectively single mode guidance, high nonlinearity and flattened, near-zero dispersion at telecom wavelengths. The use of an all solid fibre design allows for easier control of the structural parameters and better longitudinal consistency, as compared to air/glass HF counterparts. We have demonstrated that dispersion in this fibre can be controlled to the $\mathrm{ps} / \mathrm{nm} / \mathrm{km}$ level, which is unprecedented for a compound glass, high $\mathrm{y}$ fibre. The fabricated fibre presents an absolute dispersion $<5$ $\mathrm{ps} / \mathrm{nm} / \mathrm{km}$ over nearly $200 \mathrm{~nm}$, a high nonlinearity of $820 \mathrm{~W}^{-1} \mathrm{~km}^{-1}$, and a loss of 2.1 $\mathrm{dB} / \mathrm{m}$ at $1.55 \mu \mathrm{m}$. The fibre also experimentally shows the potential for efficient wideband wavelength-conversion.

This research was funded by the European Communities Seventh Framework Programme FP/2007-2013 (224547, PHASORS).

\section{References}

1 J.C.Knight et al., Opt. Letters 21, 1547 (1996).

2 W.Reeves et al., Opt. Express 10, 609 (2002).

3 J.Y.Y.Leong et al., Proc. ECOC'05, Th4.4.5 (2005).

4 Schott E-Catalog Optical Glass 2000.

5 F.Poletti et al., J. Opt. Soc. of Am. B 24, 2185 (2007).

6 T.Miya et al., IEEE J. Quan. Electron., QE-17, 858 (1981). 